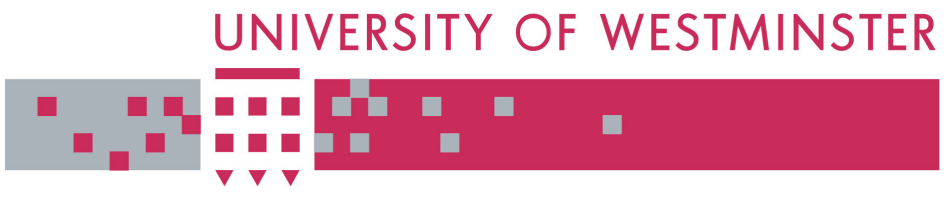

\title{
WestminsterResearch
}

http://www.wmin.ac.uk/westminsterresearch

\section{Room equalization based on iterative simple complex smoothing of acoustic impulse responses.}

\author{
Ahfir Maamar ${ }^{1}$ \\ Izzet Kale ${ }^{2}$ \\ Berkani Daoud ${ }^{3}$ \\ ${ }^{1}$ Department of Informatics, University of Laghouat \\ 2 School of Informatics, University of Westminster \\ ${ }^{3}$ Department of Electronics, ENPA, BP: 182, Alger
}

Copyright (C) [2006] IEEE. Reprinted from the proceedings of the 2006 IEEE International Conference on Acoustics, Speech and Signal Processing, ICASSP 2006, pp. V-109-V-112.

This material is posted here with permission of the IEEE. Such permission of the IEEE does not in any way imply IEEE endorsement of any of the University of Westminster's products or services. Internal or personal use of this material is permitted. However, permission to reprint/republish this material for advertising or promotional purposes or for creating new collective works for resale or redistribution must be obtained from the IEEE by writing to pubs-permissions@ieee.org. By choosing to view this document, you agree to all provisions of the copyright laws protecting it.

The WestminsterResearch online digital archive at the University of Westminster aims to make the research output of the University available to a wider audience. Copyright and Moral Rights remain with the authors and/or copyright owners.

Users are permitted to download and/or print one copy for non-commercial private study or research. Further distribution and any use of material from within this archive for profit-making enterprises or for commercial gain is strictly forbidden.

Whilst further distribution of specific materials from within this archive is forbidden, you may freely distribute the URL of the University of Westminster Eprints (http://www.wmin.ac.uk/westminsterresearch).

In case of abuse or copyright appearing without permission e-mail wattsn@wmin.ac.uk. 


\title{
ROOM EQUALIZATION BASED ON ITERATIVE SIMPLE COMPLEX SMOOTHING OF ACOUSTIC IMPULSE RESPONSES
}

\author{
Ahfir Maamar $^{(1)}$, Izzet Kale ${ }^{(2)}$ and Berkani Daoud ${ }^{(3)}$ \\ (1) Department of Informatics, University of Laghouat, BP: 37G, Laghouat, ALGERIA, Email: \\ m.ahfir@mail.lagh-univ.dz. \\ (2), Applied DSP and VLSI Research Group, Department of Electronic Systems, University of \\ Westminster, 115 New Cavendish Street, London, UK. Email: kalei@westminster.ac.uk, \\ (3) Department of Electronics, ENPA, BP: 182, Alger, ALGERIA. Email: dberkani@hotmail.com.
}

\begin{abstract}
This paper presents a room equalization method based on iterative simple complex smoothing of measured acoustic impulse responses. This is useful in cases of long duration impulse responses. Corresponding time reduced impulse responses are derived which conform to perceptual principles. The smoothed impulse responses are then used to design equalization filters. Results from an audioconferencing reverberant room using objective and subjective tests show that we can improve the measured and perceived quality of audio reproduction.
\end{abstract}

\section{INTRODUCTION}

Direct equalization of room acoustics based on inverse filtering of measured mixed-phase impulse responses introduces a number of theoretical and practical problems. Particularly, the required extremely long lengths of the inverse filters and their sensitivity to possible changes in the listener position inside the room [1], [2]. When the smoothed room impulse response was used for the design of 'inverse filters' for audio or acoustic digital equalization applications, it was found that the effect of smoothing was desirable since it was allowing the design of filters with lower sensitivity - from the perceptual point of view - to possible changes in the listener position inside the room [3], [4], [5]. The work presented here is based on the concept of complex smoothing performed by the simple case of constant bandwidth filters for inverse filter' design for acoustic impulse responses equalization. This concept is adopted because modified impulse response functions could be derived, which would present functions of reduced complexity and also be in agreement with the perceptual principles. The work presented in this paper is organized as follows: Section 2 presents the theory of the simple complex smoothing [3]. In Section 3 an equalization filter design method is proposed, which is based on iterative simple complex smoothing. Section 4 shows the magnitude equalization performance results achieved using this proposed method in the context of a real audio-conferencing room (cnet-LANNIONFRANCE).

\section{SIMPLE COMPSLEX SMOOTHING}

Let us consider a discrete-time room impulse response $h(n)$ and its discrete-frequency response $H(k)$, where $0 \leq n \leq N-1,0 \leq k \leq N-1$ and $N$ is the number of samples, representing the impulse response length. Then the complex smoothing operation for the simple case of constant bandwidth filter can be described as a circular convolution [3]:

$$
H_{c s}(k)=H(k) \otimes W_{s m}(k)
$$

where $\otimes$ denotes the operation of circular convolution and $W_{s m}(k)$ is a spectral smoothing function having the general form of a low-pass filter.

This is equivalent in the time domain to:

$$
h_{c s}(n)=h(n) N w_{s m}(n)
$$

where $w_{s m}(n)$ being the inverse DFTsequence of $W_{s m}(k)$. To consider this spectral smoothing function in a halfwindow sense for both parts of the symmetric spectrum

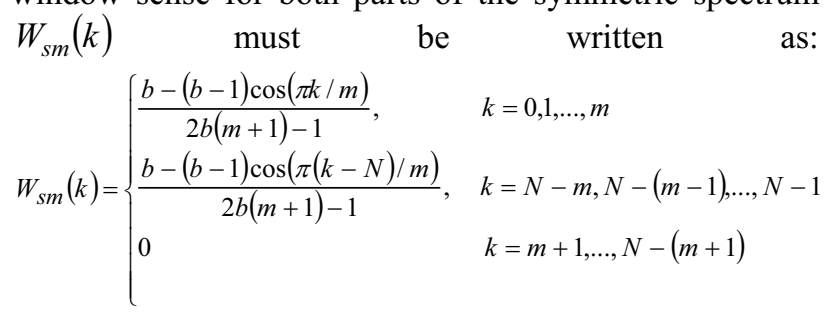

where $m$ (samples) is defined as the smoothing index corresponding to the length of the half window. When $b=1$, this function represents an ideal low-pass filter (rectangular frequency smoothing function),

$$
W_{s m}(k)= \begin{cases}\frac{1}{2 m+1}, & k \in\{0,1, \ldots, m\} \cup\{N-m, N-(m-1), \ldots, N-1\} \\ 0 & k \in\{m+1, \ldots, N-(m+1)\}\end{cases}
$$


In this case the corresponding time window function which is represented with a sinc function $w_{s m}(n)$ can be evaluated easily as:

$$
w_{s m}(n)=\frac{1}{N} \frac{\sin (\pi n(2 m+1) / N)}{\pi n(2 m+1) / N}
$$

Given that $H(k)$ is a complex function; in general $W_{s m}(k)$ should be a complex function. However, the preceding expressions represent it as a real function, assuming it to be a zero-phase function. This assumption was adopted because of physical considerations, since with smoothing it is required to avoid imposition of any unwanted effects on the phase of the original function and it is also desirable to maintain the half-window time profile appropriate for capturing transient data of the acoustic impulse response that are significant to the listener (direct signal and first reflections).

\section{EQUALIZATION FILTER DESIGN BASED ON ITERATIVE SIMPLE COMPLEX SMOOTHING}

In order to overcome the known problems of direct equalization as stated in the introduction, we propose in this section an equalization method based on iterative simple complex smoothing of room impulse responses. In this method the original room impulse response $h(n)$ is assumed to be the result of smoothing operation in the time domain of an initial one (its length was $2 N$ ) by a half smoothing window $w_{s m}(n)$ of $N$ samples. Then in the first iteration $h(n)$ will be halved in length after the application of a half smoothing window $w_{s m}(n)$ of $\frac{N}{2}$ samples. As a result a reduced-length complex smoothed impulse response $h_{c s}(n)$ will be produced and a minimum-phase inverse impulse response $g_{c s, m p}(n)$ for smoothed response magnitude equalization will be evaluated by using the homomorphic method [6], since the smoothed response may present a mixed-phase character. This minimumphase inverse impulse response $g_{c s, m p}(n)$ will be the 'equalization filter', employed on the original acoustic impulse response $h(n)$ according to the following expression:

$$
h_{e q}(n)=h(n) \otimes g_{c s, m p}(n)
$$

The iteration process is repeated only if the result of the smoothing operation will be meaningful, that is, the early time components (direct signal and first reflections) of the already smoothed impulse response $h_{c s}(n)$ must be preserved when halving it again with a half smoothing window $w_{s m}(n)$. The procedure may be stated as follows:

1. Set the iteration index $i$ to 0 and choose the parameters $b$ and $m$.

2. Compute the spectral smoothing window

$$
W_{s m}(k) \text { using (3) for } k=0,1,2, \ldots, \frac{N}{2^{i}} \text {. }
$$

3. Compute the corresponding time smoothing window $w_{s m}(n)$ by using the IDFT and apply it for $n=0,1,2, \ldots ., \frac{N}{2^{i+1}}$.

4. Compute the smoothed impulse response $h_{s m}{ }^{i+1}(n)$ as:

$$
h_{c s}{ }^{i+1}(n)=h_{c s}{ }^{i}(n) \frac{N}{2^{i}} w_{s m}(n)
$$

where $h_{c s}{ }^{0}(n)=h(n)$.

5. Evaluate the corresponding minimum-phase inverse impulse response $g_{c s, m p}{ }^{i+1}(n)$ using the homomorphic method

6. Compute the equalized room impulse response $h_{e q}(n)$ as:

$$
h_{e q}(n)=h(n) \otimes g_{c s, m p}^{i+1}(n)
$$

7. Increment $i, i=i+1$.

\section{RESULTS}

The proposed method was applied to a measured acoustic impulse response of an audio-conferencing room at a sampling frequency of $16 \mathrm{kHz}$ and recorded to $N=8192$ (Figure 1) [8]. In this example three iterations $(i=0,1,2)$ of the smoothing process using an initial half smoothing window (Figure $2-b=0.5$ and $m=3$ ) was needed to preserve the initial transient portion of the acoustic impulse response. A reduced-length of the smoothed response $h_{c s}{ }^{3}(n)$ (Figure 3) was produced and then used to construct the appropriate minimum-phase inverse impulse response $g_{c s, m p}{ }^{3}(n)$ by using an iterative version of the homomorphic method [7] with an optimum number of iterations $L=8$. This means that $g_{c s, m p}{ }^{3}(n)$ is constructed by 8 successive convolutions of partially calculated minimum-phase inverse impulse responses $g_{c s, m p}{ }^{1 / 8}(n)$

(Figure 4). The particular choice of this iterative version over the standard method is because of reduced time domain aliasing given a fixed number $N$ for DFT computation.

In order to assess the performance of our algorithm, we used an objective frequency domain error criterion, which estimates the standard deviation of the magnitude response from a constant level [2]. The error criterion $\Delta(d B)$ is given as follows:

$$
\Delta=\left[\frac{1}{N} \sum_{k=0}^{N-1}\left(10 \log _{10}|H(k)|-H_{m}\right)^{2}\right]^{1 / 2}
$$

where:

$$
H_{m}=\frac{1}{N} \sum_{k=0}^{N-1} 10 \log _{10}|H(k)|
$$

and evaluated for both the original and equalized responses. This error criterion was also evaluated for both 
a second acoustic impulse response which is different to the initial one, and its corresponding equalized response using the same inverse filter used to equalize the initial one; assessing in this way potential robustness of the proposed method to possible changes in the listener position inside the room.

The results for magnitude equalization are tabulated below in the form of the objective error criterion evaluated for both original and equalized responses. The magnitude responses of the two cases (initial and second) for both original and equalized are shown in Figure 5.

Table 1. Objective error criterion (dB) evaluated for both original and equalized responses.

\begin{tabular}{|c|c|c|}
\hline $\begin{array}{c}\text { Acoustic } \\
\text { Response }\end{array}$ & $\begin{array}{c}\text { Original } \\
\text { Response }(\mathrm{dB})\end{array}$ & $\begin{array}{c}\text { Equalized } \\
\text { Response }(\mathrm{dB})\end{array}$ \\
\hline Initial & 8.31 & 3.54 \\
\hline Second & 7.46 & 5.26 \\
\hline
\end{tabular}

These results indicate a significant reduction of the objective error criterion as it can be seen as spectral magnitude deviation from flatness for both cases (Figure 5). The subjective testing was also evaluated by listening tests through headphones using Matlab's Audio Tools and a reverberated anechoic speech as the original signal test. The subjective results indicated a significant perceptual preference for the equalized signal over the original. These results can be considered as promising for the case of possible changes in the listener position inside the room.

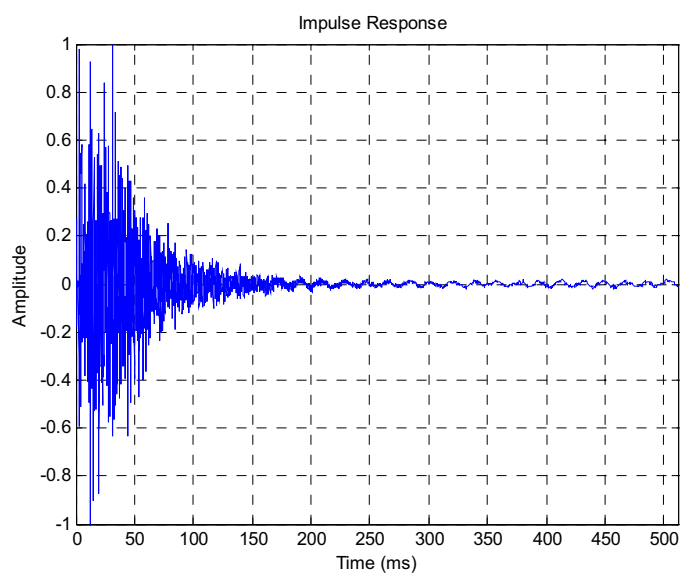

Figure 1. Measured acoustic impulse response

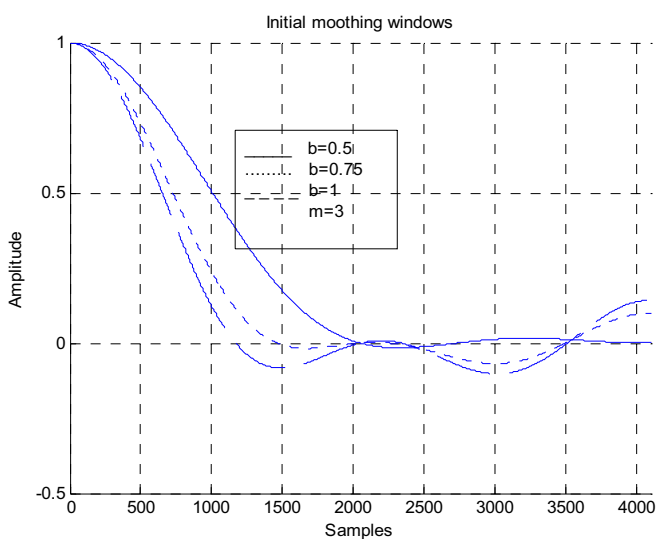

Figure 2. Initial smoothing windows for $i=0$

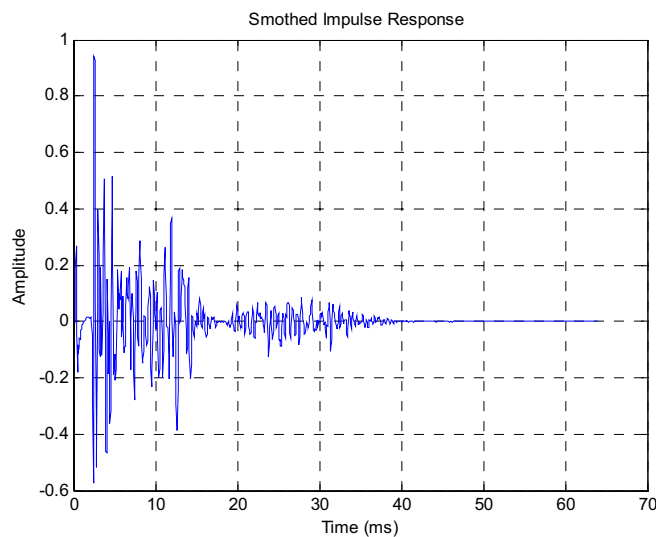

Figure 3. Smoothed impulse response $h_{c s} 3(n)$

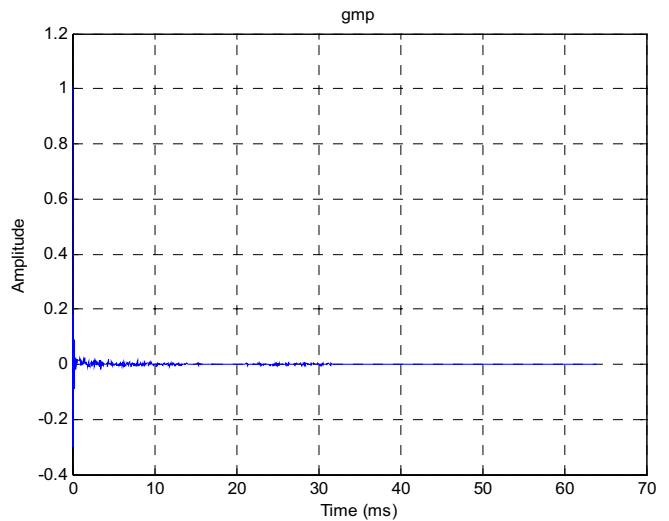

Figure 4. Partial minimum-phase inverse impulse response $g_{c s, m p}{ }^{1 / 8}(n)$ calculated using the iterative version of the homomorphic method with $L=8$. 


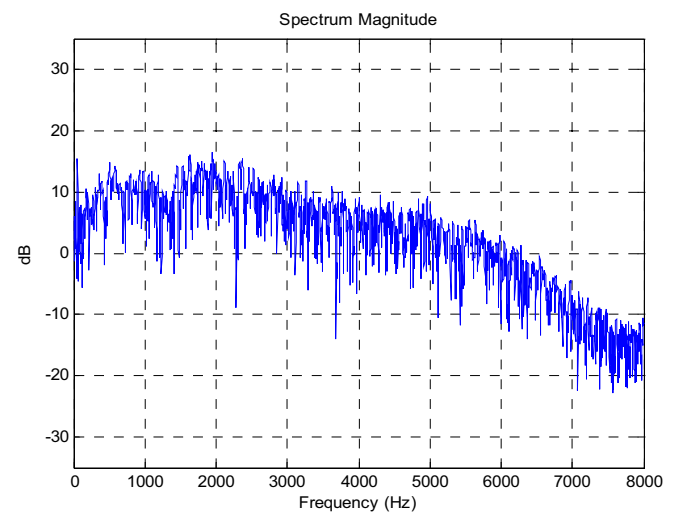

(a)

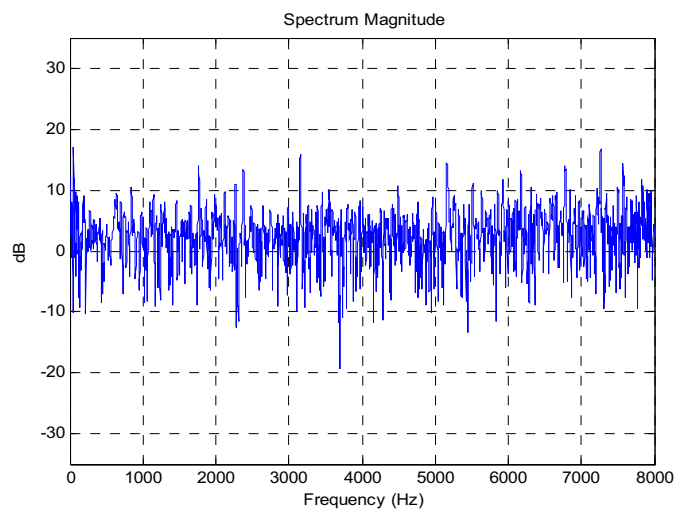

(b)

Figure 5-1. Deviation of magnitude response from flatness (a) Initial original response, b) Equalized response

\section{CONCLUSIONS}

Equalization filters design based on iterative simple complex smoothing has been proposed for room equalization. The algorithm can be useful in cases of long duration acoustic impulse responses since it results in impulse responses of reduced complexity which preserve the initial transient data that are significant to the listener. The algorithm also gives the possibility of stopping the smoothing process when the corresponding equalization filter produces some pre-determined equalized magnitude response.

\section{REFERENCES}

[1] B. D. Radlovic and R. A. Kennedy, "Non-minimum phase equalization and its subjective importance in room acoustics", IEEE Transactions on Speech and Audio Processing, vol. 8, no. 6, Nov 2000.

[2] J. N. Mourjopoulos, "Digital Equalization of Room Acoustics", J. Audio. Eng. Soc, Vol 42, N¹1, 1994 November.

[3] P.Hatziantonio and J.Mourjoupoulos, " Generalized Fractional-Octave Smoothing of Audio and Acoustic Responses", J. Audio. Eng. Soc, vol 48, No 4, pp 259-280, 2000 April.

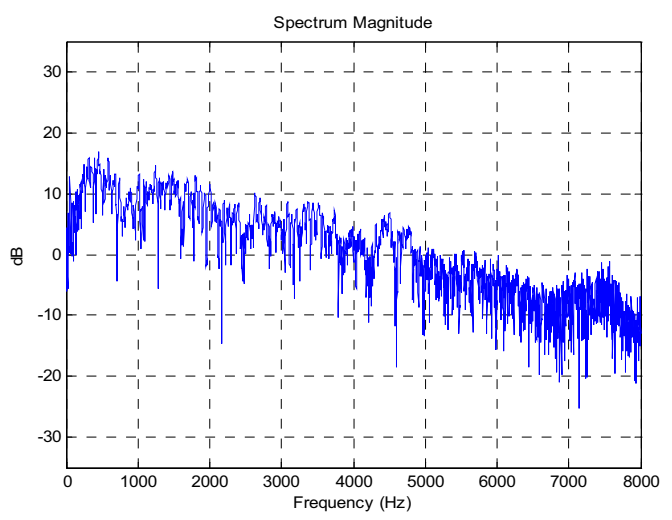

(a)

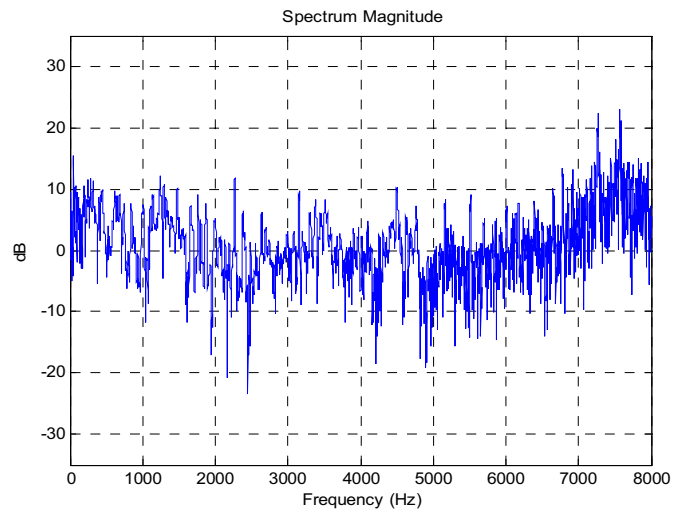

(b)

Figure 5-2. Deviation of magnitude response from flatness a) Second original response, b) Equalized response

[4] P. M .Clarkson, J.Mourjopoulos and J.K.Hammond, "Spectral, Phase and Transient Equalization for Audio Systems", J. Audio. Eng. Soc, vol 33, pp 127-132, 1985 Mar.

[5] S. Salamouris, K. Politopoulos, V. Tsakiris and J. Mourjopoulos, "Digital System for Loudspeaker and Room Equalization ", presented at the $98^{\text {th }}$ Convention of the Audio Engineering Society, J. Audio. Eng. Soc, (Abstracts), vol 43, pp 396, 1985 May.

[6] Neely S. T. and J. B. Allen, "Invertibility of a room impulse response", J. Acoust. Soc. Am, vol. 66, no. 1, pp. 165-169, July 1979.

[7] B. D. Radlovic and R. A. Kennedy, "Iterative cepstrumbased approach for speech dereverberation ", ISSPA' 99, Brisbane, Australia, 22-25 August 1999.

[8] J.P.Jullien, A.Gilloire and A.Saliou, "Mesure des reponses impulsionnelles en acoustique", Note technique NT/LAA/TSS/181, Novembre 1984. 